\title{
UNUSUAL LONGEVITY IN ANEURYSM OF THE THORACIC AORTA
}

\author{
BY \\ RALPH KAUNTZE \\ From St. George's Hospital \\ Received June 24, 1946
}

\begin{abstract}
A healthy looking man, aged 36, complaining of recurrent streaking of the sputum with blood and of difficulty in swallowing solids during the previous month was admitted to St. George's Hospital under the care of Dr. F. Golla in 1921. In addition, there had been pains in the back of the head and left side of the neck, which had worried him on occasion since 1919; latterly numbness of the left arm had been noted.
\end{abstract}

From 1900 to 1919 he had served in the regular Army, contracting enteric and malaria in 1901 while in South Africa. Since 1919 he had been employed as a carman, a not exceptionally strenuous occupation. He had one son alive and well, and his wife had had no miscarriages.

Examination at this time revealed visible pulsation above the manubrium sterni, a tracheal tug, and pulse inequality, the left radial being variable in volume but always weaker than the right. The apex beat was four and a half inches from the mid-line in the fifth left interspace; there was a ringing aortic second sound and an aortic systolic murmur conducted upwards; the systolic blood pressure in the right arm was $132 \mathrm{~mm}$. (that in the left arm was later recorded as 80 compared with 112 in the right arm). There was a systolic murmur over the lower cervical spines and dullness at both lung apices in front, and inequality of the pupils.

The blood Wassermann reaction was positive. The date of primary infection is not known; although his earlier notes give 1900 , this is now denied by the patient with some vehemence.

A radiograph with screening was reported as showing a sacculated mass to the left of the aorta in which pulsation was seen, thereby confirming a clinical diagnosis of aortic aneurysm. During this period in hospital his general condition continued poor, the sputum was repeatedly blood-tinged, and at times there was orthopnœa.

From September to December 1921 he received potassium iodide orally and mercury by inunction. In December $0.35 \mathrm{~g}$. of novarsenobenzol was given intravenously, and in the succeeding four weeks a further $2.5 \mathrm{~g}$. His condition at discharge, on January 21, 1922, was noted as " little changed," the sputum being intermittently tinged with blood and the neck pain persisting.

During the subsequent eight months he was confined to bed, after which he slowly started to get about, the sputum being no longer blood-stained. Although he did not attempt any regular work, he remained reasonably well until September 1931 when he again attended St. George's Hospital complaining of giddiness and pains in the chest. There had not been further anti-syphilitic treatment since 1922.

In July 1932 after two short courses of sulphostab and bismuth injections the blood Wassermann reaction was negative. Bismuth injections and mercury by mouth were given at intervals up to 1940 , when the blood Wassermann reaction was again negative and the Kahn reaction 
positive. Meanwhile, in 1933, he successfully recovered from an operation for acute appendicitis, and in 1934 from an attack of jaundice; at this latter occasion a radiograph showed " a saccular aneurysm with calcification."

In 1941 there were again small hæmoptyses throughout the year. The aneurysm increased in size between 1940 and 1942, when a swelling was noted between the top of the manubrium and the angle of Louis. In April 1944 there was sudden onset of aphasia, possibly embolic in origin, from which recovery has not been complete.

In May 1946 he was admitted to St. George's Hospital under the care of Dr. C. B. Levick, after a sudden increase in the size of the aneurysm; during the previous three weeks there had been weakness of the right arm and forearm, of gradual onset. Occipital and cervical pains were still at times troublesome. The initial symptom, and one that has been present intermittently throughout, was pain in the neck and occiput. The significance of this referred pain in the diagnosis of aneurysm of the aortic arch was emphasized by Graham Steell (1911).

Examination showed slight cyanosis of the lips, a pulsatile swelling four inches transversely

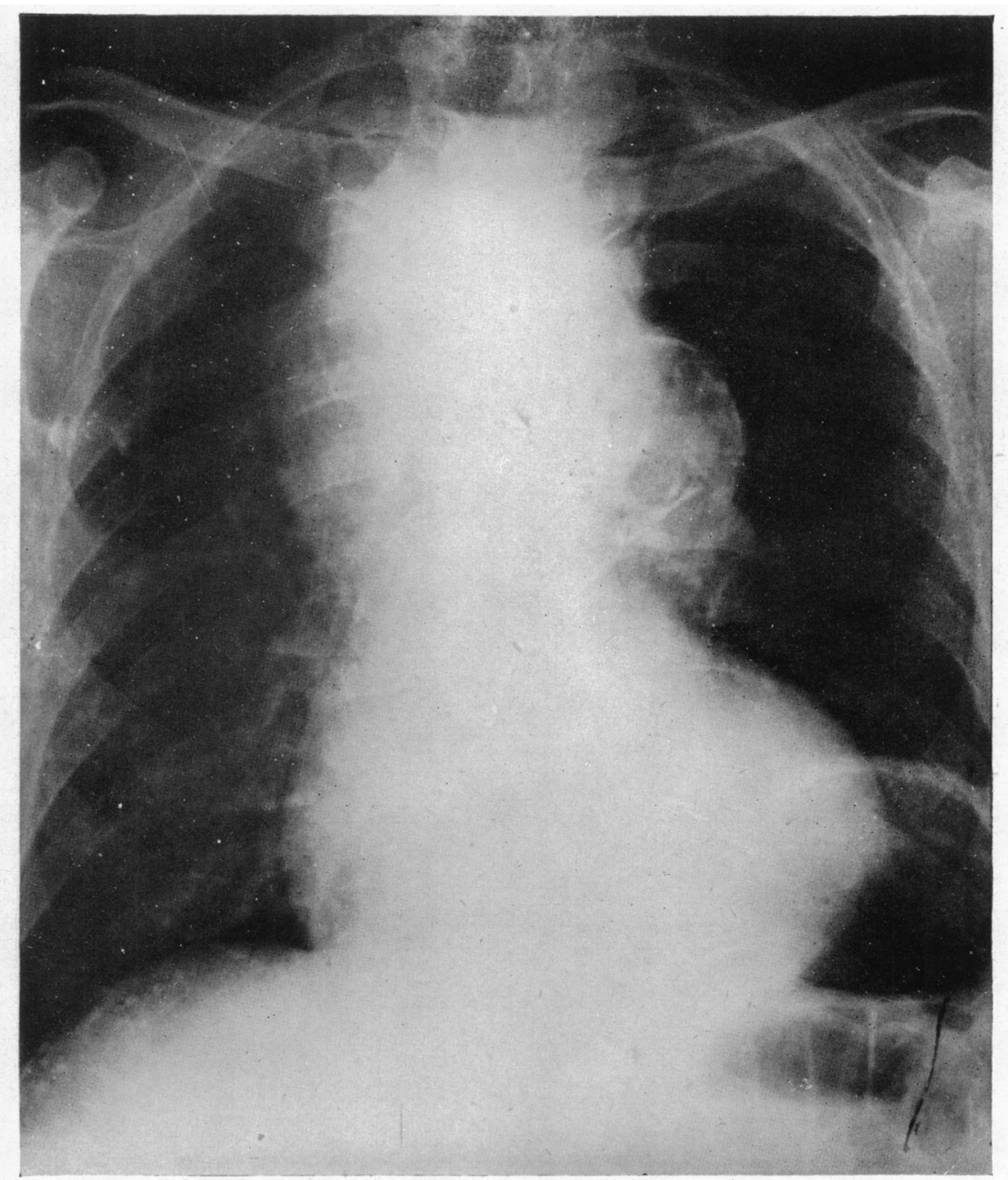

FIG. 1.-X ray of the aneurysm with calcification in the aorta. May 1946. 
by three and a half inches longitudinally occupying the whole area of the manubrium, with a weaker and more expansile area in the right periphery; there was no discoloration of the overlying skin.

The pulse was collapsing in type, rate 65-70, the left radial pulse being weaker and lagging behind the right, the pulse inequality being little altered by forced inspiration or expiration. There was dullness from below the right clavicle to the fifth rib extending two inches to the right of the sternum, the apex beat was four inches out in the fifth left interspace, and there were soft aortic systolic and diastolic murmurs: B.P. right arm 175/70, left 155/65.

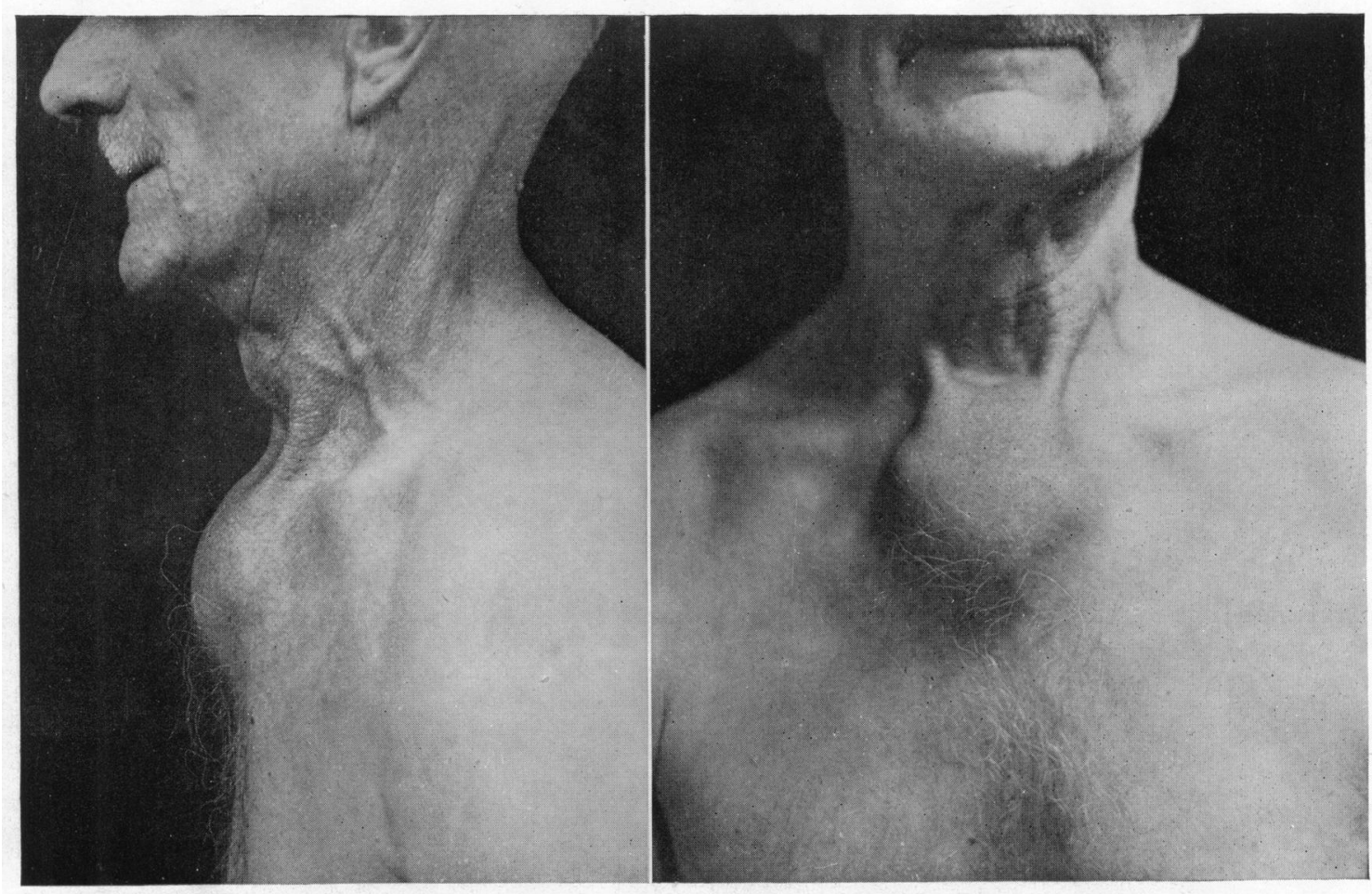

Fig. 2.-Lateral and anterior views of the aneurysm presenting through the manubrium. May 1946.

There were motor aphasia, moderate weakness of all movements of right arm and forearm particularly noticeable in skilled actions, slight wasting of these muscles, hypoalgesia over a small area of the flexor surface of the right forearm, but no positional loss. The abdominal reflexes were absent, the tendon reflexes equal except for ankle jerks which were not elicited, the plantar responses flexor, and the pupils regular, equal, and reacting. There was moderate arteriosclerosis of the retinal vessels and left vitreous opacities. The vocal cords showed normal movements. The weakness of the right arm seemed probably the result of a cortical thrombosis.

X-ray examination showed an aneurysm of the aortic arch, with calcified plaques in the aorta and in parts of the aneurysmal sac; left ventricular hypertrophy; trachea displaced to the right; left diaphragm raised, ? phrenic palsy. By May 15, 1946, a small increase in the size of the visible portion of the aneurysm had occurred, since when there has been little change and equilibrium appears to have been restored. The occipital and neck pains are not now evident, there is no dysphagia, and there is little complaint. 


\section{DisCUSSION}

The first symptoms referable to the aneurysm occurred in 1919, and in 1921 the lesion was sufficiently advanced to cause dysphagia and hæmoptysis, so that the probable duration in this aneurysm is not less than 27 years. Colt (1926) from a consideration of 482 cases of saccular aneurysm of the thoracic aorta in men found the average duration of life from the onset of symptoms 19 months; among 72 women the duration was 22 months. The expectation of life in aneurysm of the ascending or transverse aorta amongst men with onset at 60 years was rather less than twice that with onset at 35 years: whereas expectation with aneurysm of the descending aorta was the reverse of this.

De Havilland Hall (1913) in 27 private cases gives the duration after diagnosis as 32 months, and suggests that three years represents the average expectation from first symptom; he also mentions an instance of survival for 15 years in a builder's foreman.

Herz (1919) noted a patient, aged 57, with aneurysm of the ascending aorta of 16 years' duration, still alive at the time of the report. Stewart and Garland (1932) record a patient with saccular aneurysm of the aortic arch, first diagnosed at the age of 33, who lived for a further 29 years during which he enjoyed good health, working as a janitor; eventually death resulted from a hypernephroma: he had received iodides for two months and then no further treatment.

\section{SUMMARY}

A case of aneurysm of the thoracic aorta of prolonged duration is recorded.

In 1921 the aneurysm was "weeping," but by the end of 1922 the patient was fairly well. Hæmoptysis, after being present in 1921, recurred temporarily in 1934 and again in 1941.

In 1921 treatment consisted of mercury inunctions, potassium iodide, and later five injections of novarsenobenzol, following which there was no further anti-syphilitic treatment until September 1931.

In 1946 his condition is still reasonably good.

I wish to thank Dr. C. B. Levick for permission to publish this case.

Colt, G. H. (1926-27). Quart. J. Med., 20, 331-348.

Hall, F. de H. (1913). Lancet, 1, 803, 869, 945.

Herz, L. F. (1919). Med. Rev. of Rev., N.Y., 25, 476.

Steell, G. (1911). Lancet, 2, 1606.

Stewart, M. J., and Garland, H. G. (1932). Ibid., 2, 570. 